\title{
Atypical manifestations of malaria
}

This article was published in the following Dove Press journal:

Research and Reports in Tropical Medicine

19 January 2011

Number of times this article has been viewed

\author{
Syed Ahmed Zaki \\ Preeti Shanbag \\ Department of Pediatrics, Lokmanya \\ Tilak Municipal Medical College \\ and General Hospital, Sion, Mumbai, \\ Maharashtra, India
}

\begin{abstract}
Malaria remains a major cause of morbidity and mortality worldwide. The classic presentation of malaria with paroxysms of fever is seen only in $50 \%-70 \%$ of patients. Development of immunity, increasing resistance to antimalarial drugs, and indiscriminate use of antimalarial drugs have led to malaria presenting with unusual features. This review compiles a description of the various atypical manifestations of malaria.
\end{abstract}

Keywords: malaria, atypical manifestations

\section{Introduction}

Despite intensive efforts over the last century to understand and control malaria, it remains a leading cause of morbidity and mortality in humans. An estimated 300-500 million people contract malaria each year, resulting in 1.5-2.7 million deaths annually. ${ }^{1,2}$ Malaria is caused by intraerythrocytic protozoa of the genus Plasmodium, with humans being infected by one or more of the following species: Plasmodium falciparum, Plasmodium vivax, Plasmodium ovale, Plasmodium malariae, and Plasmodium knowlesi. Plasmodia are primarily transmitted by the bite of an infected female Anopheles mosquito. Infections can also occur through exposure to infected blood products and by congenital transmission. ${ }^{2}$ P. falciparum is responsible for the majority of severe and fatal malaria. ${ }^{3}$ As the term 'benign tertian malaria' implies, vivax malaria is usually an uncomplicated disease that runs a benign course and is rarely fatal. ${ }^{4}$ This clinical paradigm has been challenged recently by numerous reports of symptoms and signs of severe disease, and even deaths due to $P$. vivax monoinfections. ${ }^{4-10}$

The classic presentation of malaria consists of paroxysms of fever alternating with periods of fatigue but otherwise relative wellness. Symptoms associated with febrile paroxysms include high fever, rigors, sweats, and headache, as well as myalgia, back pain, abdominal pain, nausea, vomiting, diarrhea, pallor, and jaundice. ${ }^{2}$ However, classical presentation is seen in only $50 \%-70 \%$ of the cases with the rest having atypical manifestations. In endemic regions, malaria can present with unusual features due to development of immunity, increasing resistance to antimalarial drugs, and the indiscriminate use of antimalarial drugs. ${ }^{11}$ As a result of lack of awareness of atypical manifestations, it is not uncommon for malaria to get diagnosed late or even remain unrecognized, resulting in severe illness or death. We briefly review the atypical manifestations of malaria.
Correspondence: Preeti Shanbag 80I, Yashowan, TH Kataria Marg, Mahim, Mumbai 400016, Maharashtra, India

$\mathrm{Tel}+9$ I 222407638 I

Fax +9| 2224086100

Email pshanbag@gmail.com 


\section{Review methods and literature search}

We systematically conducted a bibliographic search using the National Library of Medicine via PubMed and Medline search engines of relevant published English literature from 1960 to 2002. Key phrases used included malaria and the following terms: pathophysiology, atypical manifestations, hepatic, splenic, hepatobiliary, gastrointestinal, metabolic, renal, neurological, psychiatry, cerebellum, and neurological sequelae; skin, ocular, musculoskeletal, hematological, respiratory, cardiovascular, endocrine, pregnancy, and malnutrition. Abstracts were reviewed for relevance, and pertinent articles were reviewed in full. We searched article bibliographies to identify further relevant work. We also reviewed the chapters on malaria in the standard textbooks of pediatrics, medicine, and infectious diseases.

\section{Brief overview of the pathophysiology in malaria}

The development of severe malaria probably results from a combination of parasite-specific factors, such as adhesion and sequestration in the vasculature and the release of bio-active molecules, together with host inflammatory responses. The sequestration of red cells containing mature forms of the parasite (trophozoites and meronts) in the microvasculature is believed to cause the major complications of falciparum malaria, particularly cerebral malaria. ${ }^{12}$ The sequestration of parasitized red blood cells (PRBCs) in the relatively hypoxic venous beds allows optimal parasite growth and prevents the PRBCs from being destroyed by the spleen. Sequestration is believed to be a specific interaction between PRBCs and the vascular endothelium. The adhesion of the PRBCs to the vascular endothelium (cytoadherence) reduces the microvascular blood flow, which may explain organ and tissue dysfunction such as coma. The adherence of nonparasitized RBCs (NPRBCs) to PRBCs (rosetting) and PRBCs to PRBCs (agglutination) have also been implicated in the pathogenesis of cerebral malaria. As the parasite grows within the RBCs, the erythrocyte becomes less deformable and may contribute to the RBC destruction and impair the microcirculatory flow. The reduction in red cell deformability occurs not only in PRBCs, but also in the NPRBCs. The NPRBCs have to undergo considerable deformation as they squeeze through the sequestered microcirculation. Microvascular perfusion in severe falciparum malaria is, therefore, limited by mechanical obstruction, adherence of other RBCs, and the stiffness of the nonadherent RBCs. Blood concentrations of proinflammatory cytokines are raised in cerebral malaria, as in many severe infections in which inflammasome is involved. Tumor necrosis factor $\alpha$ (TNF- $\alpha$ ) upregulates endothelial cytoadherence receptors and can cause hypoglycemia and dyserythropoiesis, which are features of severe disease. ${ }^{12-17}$ Compared to the pathophysiology of falciparum malaria, there are large gaps in knowledge for vivax malaria. Because $P$. vivax preferentially infects young RBCs, parasitemias rarely exceed $2 \%$ of circulating RBCs, and high parasite burdens are not a feature of severe disease. Because all stages of $P$. vivax are visible in peripheral blood, P. vivax is not believed to sequester or cause end-organ dysfunction. However, cytokine production during $P$. vivax infections is higher than P. falciparum infections of similar parasite biomass. ${ }^{14}$

\section{Atypical neurological manifestations of malaria Cerebral malaria}

Cerebral malaria is one of the most common and potentially life-threatening complications of $P$. falciparum malaria and is characterized by unarousable coma. ${ }^{18}$ However, coma associated with $P$. vivax is rare, and its etiology is the least characterized of the syndromes associated with $P$. vivax. Cytoadherence phenomena are believed to be central to the etiology in falciparum malaria, but their role in P. vivax malaria remains unclear. Potential factors suggested are the presence of concurrent infections, mixed plasmodium infections, reversible local changes in the microvasculature, endothelial activation, and injury and microvascular thromboinflammatory responses. ${ }^{14}$ Convulsions are common, especially in children. ${ }^{18-21}$ Most seizures seem to be generalized, but electroencephalogram identifies a focal origin in many patients. ${ }^{18,21}$ Malarial encephalopathy is usually symmetric with localizing signs being noted infrequently. Neurological signs include depressed sensorium, convulsions, passive resistance to neck flexion with divergent gaze, sixth nerve palsy, absence of abdominal reflexes, extensor plantars with variable tone, and deep tendon reflexes. ${ }^{18,20,21}$ Generalized hypertonia and opisthotonus may be observed in severe cases, suggesting brain stem dysfunction. Some signs (used in prognostic calculation) in children predict poor outcome. These include deep coma, decerebration, absence of corneal reflexes, convulsions on admission, and age $<3$ years. ${ }^{20}$

A variety of neuro-ophthalmological signs may be noted. Dysconjugate gaze is a common finding. ${ }^{21}$ Corneal and conjunctival reflexes are usually intact with symmetric pupils reacting normally to light. Papilledema, although rare, is a poor prognostic sign. ${ }^{18}$ Retinal hemorrhages and unusual 
retinal 'whitening' may be observed. ${ }^{18,20,21}$ The prognosis of cerebral malaria worsens considerably with coexistent renal failure, severe jaundice, or metabolic acidosis. ${ }^{18}$

Subarachnoid hemorrhages have been described in patients with cerebral malaria. They usually occur due to the rupture of small vessels which get plugged by red cells in combination with severe thrombocytopenia and associated disseminated intravascular coagulation. ${ }^{22,23}$

Other reported atypical neurological manifestations associated with cerebral malaria include central pontine myelinosis (CPM) and spontaneous subdural empyema. ${ }^{24,25}$ $\mathrm{CPM}$ occurs in cerebral malaria as a result of ischemia and the toxic effects of the PRBCs in the cerebral microvasculature leading to capillary occlusion and damage. ${ }^{25}$

\section{Psychiatric manifestations}

Psychiatric manifestations have been described as part of cerebral malaria or after recovery from coma. ${ }^{16,18,21}$ Confusional states, delirium with hallucinations and transient amnesia, dementia, personality disturbances, and schizophrenia have been described. Agitation and confusion may develop after patient has recovered from coma. Psychiatric manifestations may be a presenting feature in patients with uncomplicated malaria in association with hyperpyrexia. ${ }^{16,18,21,26-28}$ Neuropsychiatric manifestations may also be caused by antimalarial drugs. ${ }^{29,30}$

\section{Cerebellar ataxia}

Cerebellar ataxia occurs in malaria due to extensive damage to the Purkinje cells of the cerebellum associated with hemorrhages, small infarction, and microglial infiltration. The prognosis is generally good without neurological deficit on follow-up. ${ }^{31-33}$

\section{Postmalaria neurological syndrome}

Postmalaria neurological syndrome (PMNS) is defined as the acute onset of confusion, epileptic seizures, or any other neurological or psychiatric sign occurring with a latency of several days to weeks (generally within 2 months) after an episode of successfully treated $P$. falciparum malaria. ${ }^{34}$ Schnorf and others have divided PMNS into three subtypes: a mild and localized encephalopathy affecting the cerebellum and causing delayed cerebellar ataxia, a diffuse but not severe encephalopathy causing confusion with or without epileptic seizures, and a severe generalized encephalopathy resembling an acute disseminated encephalomyelitis, with usually a good response to steroid therapy. ${ }^{35,36}$ The pathogenesis of PMNS is unknown. It could be mediated immunologically as steroids are effective in some patients. In mild cases, symptomatic treatment can result in spontaneous and favorable evolution, but in severe cases, corticosteroids are required to limit brain inflammation. ${ }^{35-37}$

\section{Guillain-Barré syndrome}

Guillain-Barré syndrome (GBS) associated with malarial infection has been reported. ${ }^{38,39}$ The exact pathogenesis of GBS following malaria infection is not known, but it is likely to be immunological. Another mechanism suggested for the development of polyneuropathy includes parasitic emboli obstructing vasa nervosum and causing anoxemic stagnation, leading to temporary demyelination. Complete recovery after disappearance of parasitemia and establishment of normal blood flow in vasa nervosum have been reported. Release of neurotoxins, associated metabolic and nutritional disturbance, immune-mediated capillary damage, and release of free radicals and TNF may also be responsible for the pathogenesis of GBS after $P$. falciparum infection. ${ }^{16,38-40}$

\section{Isolated hemiparesis}

Kochar et al have described a case of isolated hemiparesis in a 15 -year-old female patient with falciparum malaria. ${ }^{41}$ The patient was conscious; hence, the hemiparesis was not associated with cerebral malaria. Computed tomography (CT) scan of the brain was suggestive of nonenhancing hypodense area in left frontoparietal region suggestive of infarction or vasogenic edema. The patient improved within 2 months with no neurological sequelae.

\section{Neurological sequelae}

Despite adequate treatment, 3\%-29\% of survivors of cerebral malaria develop neurological sequelae in the form of psychosis, ataxia, hemiplegia, cortical blindness aphasia, pseudobulbar palsy, hearing defects, and extrapyramidal syndrome. ${ }^{18,21}$ These sequelae are more common in children. Some are transient, whereas others often improve over months, although they may not completely resolve. ${ }^{21}$ Van Hensbroek et al observed sequelae in $23.3 \%$ of children at discharge, which decreased to $4.4 \%$ at follow-up 6 months later. Cognitive and behavioral abnormalities were observed in $2 \%$ of survivors when assessed at 1 month. Depth and duration of coma and multiple convulsions were independent risk factors for these sequelae. ${ }^{42}$ Kochar et al in their study of 441 patients with cerebral malaria noted the following neurological sequelae in survivors: psychosis $(5.06 \%)$, cerebellar ataxia (4.72\%), hemiplegia (1.68\%), extrapyramidal rigidity (EPR) $(1.35 \%)$, peripheral neuropathy $(1.01 \%)$, 
EPR with trismus $(0.33 \%)$, and isolated sixth nerve palsy $(0.33 \%)$. All patients showed complete recovery on followup..$^{43}$ Epilepsy can occur as a late complication of cerebral malaria. ${ }^{18,21,44,45}$ Generalized tonic-clonic seizures as well as partial motor seizures have been reported. Several pathogenic mechanisms have been proposed: vascular/ischemic mechanism, neurotoxic effects, 'malaric granulomas of Durck', genetic mechanisms, and antibodies against voltage-gated channels. ${ }^{44,45}$

\section{Atypical ocular manifestations of malaria}

Ophthalmic manifestations described in $P$. falciparum malaria include retinal hemorrhages and edema, subconjunctival hemorrhage, retinal whitening, keratits, uveitis, papilledema, disc pallor, and vitreous hemorrhage. Patients can present with sudden onset of visual loss caused by vitreous hemorrhages, cortical blindness, or retrobulbar neuritis. ${ }^{46,47}$ Kale et al described a patient with $P$. falciparum malaria who developed retrobulbar neuritis and sudden blindness. ${ }^{48}$ Although the precise mechanism is not known, retrobulbar neuritis possibly results from damage to optic nerve fibers as a result of tissue hypoxia. The hypoxia results from sequestration of RBCs, cytoadhesion, and rosette formation, leading to decreased blood flow. Antimalarials and steroids are recommended for the treatment of this complication.

\section{Atypical musculoskeletal manifestations of malaria}

Musculoskeletal manifestations reported with malaria include rhabdomyolysis, myositis, and periodic paralysis. ${ }^{49-51}$ Miller et al postulated that TNF- $\alpha$ (myotoxin), red-cell sequestration in skeletal muscle, toxins derived from the parasite, and lactic acidosis may cause myositis, myonecrosis, and rhabdomyolysis. Early recognition of this complication is important as it can lead to acute renal failure (ARF) and electrolyte disturbances. ${ }^{50}$ Senanayake and Wimalawansa have reported three patients who, during febrile episodes of malaria, developed transient generalized muscular paralysis very similar to that seen in periodic paralysis. According to the authors, the transient hyperkalemia due to lysis of RBCs and the rigors causing intense muscular contraction occurring during febrile episodes of malaria precipitated the muscular paralysis in these patients. ${ }^{51}$

\section{Atypical hematological manifestations of malaria}

\section{Anemia}

Severe anemia is a common feature of $P$. falciparum malaria; however, in vivax-endemic areas, $P$. vivax is a major risk factor for severe anemia, particularly in young children. ${ }^{52}$ The low parasite biomass of $P$. vivax indicates that severe anemia is not due to destruction of infected RBCs alone. Malariotherapy studies have shown that for every infected RBC destroyed during vivax infection, 32 noninfected $\mathrm{RBCs}$ are removed from the circulation compared to the loss of 8 RBCs for every infected erythrocyte in falciparum malaria. Cytokine-related dyserythropoiesis also probably contributes to anemia. ${ }^{14}$

\section{Hemophagocytic syndrome}

Aouba et al described a 41-year-old woman admitted with fever, splenomegaly, and pancytopenia. ${ }^{53}$ Trophozoites and gametocytes of $P$. vivax were identified on blood smear. High serum ferritin, hypertriglyceridemia, and bone marrow hemophagocytosis were consistent with a diagnosis of hemophagocytic syndrome (HPS). Inappropriate and/ or excessive production of cytokines, such as TNF- $\alpha$, interferon $\gamma$, and macrophage colony-stimulating factor, resulting in monocyte activation have been observed in patients with malaria and could trigger HPS initiation. HPS usually resolves soon after treatment of malaria. However, prolonged HPS after falciparum malaria has been reported by Ohnishi et al in a 30-year-old man. The serum levels of interleukin-18 (IL-18) and TNF- $\alpha$ remained high for a long time even after eradicating the malarial parasite in the patient. The authors have hypothesized that HPS may continue independently in the presence of malaria parasites once the cytokine cascade is triggered. ${ }^{53,54}$

\section{White blood cell count and malaria}

Leucocytosis, leucopenia, and leukemoid reaction have been reported as atypical manifestations of malaria. ${ }^{55-58}$ In a study done by Sharma et al, out of 30 cases of $P$. falciparum malaria, leucocytosis and leucopenia were observed in $13.3 \%$ and $6.6 \%$ of the cases, respectively. ${ }^{57}$ Modiano et al found that among severe malaria patients, leucocytosis was associated with severe anemia, hypoglycemia, and a 3.5-fold increase in mortality. The authors concluded that leucocytosis occurs as a result of concomitant bacterial infection in severe malaria. ${ }^{58}$ Hence, from a practical point of view, in case of leukocytosis, coverage for Gram-negative bacteria should be added. 


\section{Thrombocytopenia}

Severe thrombocytopenia has been reported most commonly with $P$. falciparum. ${ }^{59}$ In recent years, many cases of vivaxassociated thrombocytopenia have also been described. A few postulated mechanisms for thrombocytopenia in malaria include macrophage activation, increased levels of cytokines and antiplatelet immunoglobulin which cause platelet destruction. Other mechanisms include oxidative stress, sequestration in non-splenic areas and pseudothrombocytopenia due to clumping of platelets. ${ }^{60} \mathrm{~A}$ rare case of idiopathic thrombocytopenic purpura triggered by $P$. vivax infection has also been described by Lacerda et al. ${ }^{61}$

\section{Cerebral venous thrombosis}

Cerebral venous sinus thrombosis has been reported in those with severe $P$. falciparum malaria. The mechanism of thrombosis is still unclear and may be multifactorial. Altered phospholipid in malaria-infected red cells causes increased von Willebrand factor and other coagulation factors. In addition, endothelial damage by malaria-infected red cells releases tissue factor and other cytokines, resulting in a hypercoagulable state..$^{62,63}$

\section{Sternal tenderness}

Mohapatra and Khan have described two patients with P. falciparum malaria presenting with sternal tenderness. ${ }^{64}$ Bone marrow examination and other tests ruled out malignancy. The authors have hypothesized that during the life cycle, trophozoites of $P$. falciparum usually disappear from the peripheral circulation and get sequestered in capillaries of internal organs where formation of schizonts occurs. In this process, liver, spleen, and bone marrow are affected. Bone marrow is affected by plugging of its capillaries, resulting in ischemia and sternal tenderness.

\section{Atypical renal manifestations of malaria Acute renal failure}

Renal involvement has been reported commonly with P. falciparum and occasionally with $P$. malariae and $P$. vivax infections. It can occur as an isolated complication or as a component of multiorgan involvement. ${ }^{65}$ The precise mechanism of ARF in malaria is not clearly known. Several hypotheses including mechanical obstruction caused by cytoadherence and sequestration of infected erythrocytes, immune-mediated glomerular pathology, alterations in the renal and systemic hemodynamics, release of cytokines, reactive oxygen intermediates, and nitric oxide have been proposed. In addition to above, restricted blood flow to the kidneys due to low intake of fluids, loss of fluids because of vomiting, and pyrexial sweating can cause dehydration and renal ischemia. ${ }^{65-67}$ Depending on the degree of renal hypoperfusion, the spectrum of manifestations varies from milder forms like prerenal azotemia to more severe forms of ischemic ARF. The hemolysis, disseminated intravascular coagulation, and rhabdomyolysis in malaria also contribute to the pathogenesis of ischemic tubular necrosis and ARF. ${ }^{68}$ Various histological pictures including acute tubular necrosis (the commonest), interstitial nephritis, and proliferative glomerulonephritis have been described in malarial ARF either alone or in combination. ${ }^{65-67}$

\section{Glomerular involvement}

Glomerular injury associated with malaria is commonly seen in the pediatric population. Autopsies have detected glomerular lesions in about $18 \%$ of patients with falciparum malaria. Mild proteinuria, microhematuria, and casts are reported in 20\%-50\% of cases ${ }^{69}$ Both nephrotic and acute nephritic syndromes have been described with falciparum and vivax malaria. ${ }^{66,67,70,71}$ The falciparum and vivax glomerulopathy are completely reversible within 2-6 weeks upon eradication of the disease. P. malariae is the established cause of chronic malarial nephropathy (quartan malarial nephropathy), although a few cases have been associated with $P$. vivax in children. This complication affects children and shows the characteristic histopathological lesion of mesangiocapillary glomerulonephritis with subendothelial immune complex deposits containing $\operatorname{IgG}, \mathrm{C} 3$, and malarial antigens. The disease presents as steroid-resistant nephrotic syndrome and progresses to renal failure even after successful eradication of the infection. ${ }^{66,67}$

\section{Hemolytic uremic syndrome}

Renal failure because of hemolytic uremic syndrome (HUS) secondary to malaria has been described..$^{72}$ The patient was treated with peritoneal dialysis, diuretics, and antibiotics. Malarial toxin (like Shiga toxin) can alter endothelial cell integrity and provoke the sequence of events, leading to extensive microvasculature damage resulting in HUS. Long-term renal complications due to HUS are less likely when associated with malaria, thereby assuring a good prognosis with prompt effective treatment with antimalarial drugs. However, in case of recurrences, alternative pathway complement deficiency should be ruled, as it accounts for nearly $50 \%$ of atypical HUS cases. 


\section{Priapism}

Priapism was described by Dogra et al in a 9-year-old boy with $P$. falciparum malaria and Sinha et al in a 14-year-old boy with $P$. vivax malaria. ${ }^{73,74}$ This was explained as probably secondary to sludging of blood in the venules and cavernous spaces.

\section{Urinary frequency}

De Silva et al have described a 70-year-old woman with P. falciparum malaria presenting with urinary frequency. ${ }^{75}$ Her urine culture was negative, and she responded to antimalarial treatment alone. According to the authors, the urinary frequency may have been due to the sequestration of the parasite in the detrusor muscle of the urinary bladder leading to bladder irritability.

\section{Atypical respiratory manifestations of malaria}

\section{Acute respiratory distress syndrome}

Alterations in pulmonary physiology in malaria include airflow obstruction, impaired ventilation, reduced gas transfer, and increased pulmonary phagocytic activity. ${ }^{76}$ Acute respiratory distress syndrome (ARDS) in malaria is likely to be a continuous spectrum from subclinical lung involvement in uncomplicated malaria to frank ARDS in severe malaria. ${ }^{77}$ ARDS is considered to be the most severe form of acute lung injury in malaria and has been reported most commonly with P. falciparum malaria. Reports of ARDS with P. vivax malaria are also being described. The prevalence of ARDS in patients with malaria as documented in various studies ranges from $2.1 \%$ to $29.1 \%{ }^{78}$ Observations from these studies and other published reports suggest that about $5 \%$ of patients with uncomplicated P. falciparum malaria and $20 \%-30 \%$ of patients with severe and complicated malaria requiring ICU admission may develop ARDS. The incidence of ARDS as a complication of malaria is lower in children than in adults. As in acute lung injury in other diseases, ARDS in malaria probably results from cytokine-related increases in alveolar permeability and altered alveolar fluid clearance. ${ }^{78}$

\section{Pulmonary edema}

Pulmonary edema has been reported by several investigators to be one of the fatal complications of P. falciparum malaria often in association with disseminated intravascular coagulation, renal failure, cerebral malaria, and shock. Fein et al emphasized that pulmonary edema in patients with P. falciparum malaria is noncardiogenic resulting from increased permeability of the alveolar-capillary membrane. ${ }^{79}$

\section{Interstitial pneumonia}

Munteis et al have described a case of interstitial pneumonia due to $P$. vivax malaria. ${ }^{80}$ With supportive treatment and antimalarials, the patient improved within 48 hours of admission. According to the authors, interstitial pulmonary involvement should be considered in patients with malaria and acute respiratory symptoms.

\section{Bronchiolitis obliterans organizing pneumonia}

Yale et al have reported a case of bronchiolitis obliterans organizing pneumonia as a pulmonary complication of P. vivax malaria. ${ }^{81} \mathrm{CT}$ scan of chest and transbronchial lung biopsy confirmed the diagnosis. The patient was started on steroids which were gradually tapered over 9 months with full clinical recovery.

Other respiratory manifestations of malaria include pleural effusion and lobar consolidation. ${ }^{82-84}$ Elevated pulmonary venous pressure secondary to venous and lymphatic obstruction by sequestration of infected RBCs, increased capillary permeability, and associated hypoalbuminemia are some of the mechanisms that have been proposed as the cause for pleural effusions.

\section{Atypical cardiovascular manifestations of malaria Conduction abnormalities, left ventricular hypokinesia, and pericardial effusion}

In a study carried out by Franzen et al, 22 patients without any previous history of cardiac disease were prospectively evaluated for cardiac involvement during acute malaria and $9 \pm 5$ months after recovery using noninvasive methods including resting electrocardiogram (ECG) and echocardiography. ${ }^{84}$ During the acute phase, ECG abnormalities were common $(5 / 22)$; pericardial effusion was found in two patients and global left ventricular hypokinesia in one patient infected with $P$. falciparum. On follow-up, the resting ECG and echocardiography had normalized in all patients, suggesting that persistent cardiac damage following malarial infection is rare. Kohli et al described a case of pericardial tamponade following P. falciparum infection. ${ }^{85}$ Urgent pericardiocentesis yielded $800 \mathrm{~mL}$ of hemorrhagic fluid which was positive for P. falciparum antigen. According to the authors, regional sequestration of parasites in the pericardium resulting in locally elevated cytokine levels lead to the isolated pericardial effusion. The patient recovered with supportive and antimalarial treatment. 
Echocardiography on follow-up was normal. Günther et al found ECG abnormalities in 23 of 161 patients with falciparum malaria. Nonspecific T-segment or T-wave alterations were found in 15 patients and conduction abnormalities in eight patients (one first-degree atrioventricular block, one left bundle branch block, one right bundle branch block, and five incomplete right bundle branch block). ${ }^{86}$

\section{Myocardial injury and malaria}

Diffuse myocardial injury can occur in severe malaria, resulting in an adverse outcome.

Myocarditis has been reported following malarial infection. Autopsy findings of the heart showed mechanical blockage of myocardial capillaries by malarial parasites and PRBCs probably leading to ischemic cardiomyopathy. Sharma et al described a 24-year-old male with chronic falciparum cardiomyopathy. ${ }^{87}$ The patient presented clinically with ischemic heart disease with congestive cardiac failure. His ECG revealed inferior wall infarction and ischemic ST-T changes. Despite receiving antimalarials, the patient deteriorated and finally died of pulmonary edema. Autopsy revealed a grossly dilated heart, and microscopic examination showed diffuse myocardial fibrosis, interstitial hemorrhages, and capillaries blocked with PRBCs. Repeated occlusion of coronary capillaries may have caused the anginal pain with ischemic changes resulting in congestive restrictive cardiomyopathy. In a study carried out by Mohapatra et al, of 110 patients with cerebral malaria, 13 (11.8\%) had myocardial injury as evidenced by raised cardiac troponin T levels. ${ }^{88}$ Of these 13 patients, 7 died within $72 \mathrm{~h}$ and 3 on 9th day of hospitalization. Autopsy revealed diffuse myocardial injury in three cases.

\section{Hypovolemia and cardiac dysfunction}

Children with severe malaria and metabolic acidosis have evidence of hypovolemia and evidence of cardiac dysfunction. Yacoub et al examined 30 children admitted with severe malaria by using portable echocardiography to assess their cardiac function and hemodynamic status on admission (day 0), day 1 , and discharge.$^{89}$ The authors noted several markers of hemodynamic compromise on admission, including severe tachycardia, low stroke volume index, and high inferior vena cava collapsibility index, which improved with subsequent readings. Overall, cardiac function assessed by ejection fraction and left myocardial performance index was mildly abnormal on admission compared with discharge. Acidotic patients had worse hemodynamic indicators, with a significantly higher inferior vena cava collapsibility index on day 0 , than nonacidotic patients plus lower stroke volume index and worse cardiac function with higher left myocardial performance index. Stroke volume index increased after first fluid bolus in $80 \%$ of children.

In addition to above, symmetrical peripheral gangrene, purpura fulminans, disseminated intravascular coagulation, toxic shock, and endomyocardial fibrosis have been described with malaria. ${ }^{90-94}$ Most of the cases of peripheral gangrene also had evidence of disseminated intravascular coagulation. In some of the cases, resolution of gangrene took place with conservative treatment, whereas in others, amputation was required. Song et al described two unusual cases of $P$. vivax malaria who presented with a clinical picture of toxic shock..$^{90}$ They were treated with antimalarial drugs alone. The authors emphasize the importance of considering the possibility of malarial infection in patients with a clinical picture resembling toxic shock if they have a travel history to malaria-endemic areas.

\section{Atypical gastrointestinal and hepatobiliary manifestations of malaria Hepatic involvement}

Hepatic dysfunction in malaria is common, but hepatic encephalopathy is unusual, and most of the patients described have had either isolated infection with $P$. falciparum or a mixed infection with $P$. vivax. ${ }^{95}$ Severe impairment of the sensorium is not unusual in severe malaria with cerebral malaria, hypoglycemia, hypoxia, and/or uremia due to renal involvement contributing to the altered mental status. Jaundice (serum bilirubin $>3 \mathrm{mg} \%$ ), hypersomnia, and inversion of the sleep rhythm and delirium were the initial hepatic manifestations in a study of 86 adult patients with malaria. Overt evidence of hepatic encephalopathy was seen in only 15 patients. Blood ammonia levels were raised in all the patients, but asterixis was observed in only nine patients. Triphasic waves, which are considered characteristic of hepatic encephalopathy, were seen in 3 out of 15 patients. ${ }^{96}$ In endemic areas, severe malaria should feature in the differential diagnosis of fulminant hepatic failure (FHF). It is important to differentiate the two since the prognosis in patients with severe hepatic injury due to malarial infection is better than FHF due to other causes. Devarbhavi et al in their study compared 25 patients with FHF with 25 patients with severe malarial hepatopathy simulating as FHF. ${ }^{97}$ The authors have suggested that in endemic areas, the presence of hepatomegaly and normal prothrombin time in the setting of FHF should be considered as suggestive of malaria and 
a peripheral blood smear should be obtained for diagnostic confirmation. Davenport reported a case of transplacental malaria in an infant having obstructive jaundice. ${ }^{98}$ Operative cholangiogram carried out at 6 weeks ruled out extrahepatic biliary atresia (EHBA). The differentiation between neonatal hepatitis and EHBA is important because surgical relief to be successful must be performed early.

\section{Splenic involvement}

Splenic rupture is a rare complication of malaria with lifethreatening complications being reported in up to $2 \%$ of cases. ${ }^{99}$ Most cases of splenic rupture occur during acute infection and during the primary attack. Chronically enlarged spleens are less vulnerable to rupture. The exact mechanism of spleen rupture in malaria is not known. This complication may occur despite appropriate antimalarial prophylaxis and treatment. Spontaneous splenic rupture is more common with $P$. vivax than $P$. falciparum malaria. ${ }^{100}$ Histopathological findings include hemozoin, parasitized and uninfected RBCs, and a massive proliferation of macrophages throughout the capillaries, venous spaces, and pulp spaces. There is congestion and dilatation of sinuses and scattered thrombosis, with focal necrosis in capillaries and splenic pulp. ${ }^{100}$ Agarwal et al described two children with falciparum malaria who had splenic infarcts. ${ }^{101}$ Other splenic complications reported in malaria include hypersplenism, ectopic spleen, torsion, cyst or abscess or hematoma formation, and hyperreactive malarial syndrome. ${ }^{99}$ Contini and Lewis have described a case of splenic abscess in a 21-year-old woman after repeated attacks of $P$. falciparum malaria. ${ }^{102}$ Hematoma and infarction of the spleen due to repeated malarial attacks may predispose to superimposed abscess formation. The overall prognosis of splenic abscesses remains discouraging, with a mortality of $13 \%-16 \%$ mainly due to delayed diagnosis and admission to a hospital.

\section{Other involvements}

Acute pancreatitis is a rare complication of malaria. There are isolated case reports highlighting pancreatic involvement in malaria. Autopsy studies have demonstrated that the small blood vessels of the pancreas are packed with PRBCs and rosettes, causing obstruction to capillary blood flow. ${ }^{103}$

Acalculous cholecystitis (ACC) is equally rare in malaria with very few cases reported in literature. The exact pathogenesis of ACC is not clearly understood. Gallbladder wall ischemia and reperfusion injury or the effects of eicosanoid proinflammatory mediators appear to be important. Moreover, the microcirculatory obstruction in the gallbladder due to the phenomenon of rosetting and cytoadherence of infected RBCs further contribute to the ischemia of gallbladder resulting in ACC. The course of the disease is usually self-limiting and cholecystectomy is not advised in uncomplicated cases. ${ }^{104}$ Cholecystectomy or cholecystostomy may be required in complicated (gangrene and perforation) and nonresolving cases.

Dass et al have described an 18-month-old female with $P$. vivax malaria presenting as subacute intestinal obstruction. ${ }^{105}$ The patient presented with vomiting, fever, and abdominal pain. X-ray, ultrasonography of the abdomen, and serum electrolytes were normal. Vomiting and abdominal pain are also known to be associated with malaria. Abdominal pain occurs due to ischemic changes in the intestine secondary to microvascular changes due to sequestered RBCs. Cases of frank perforation have also been reported. ${ }^{105}$ In endemic areas, malaria should be suspected in any febrile child presenting with an acute surgical abdomen.

Sood and Midha described a 16-year-old boy with $P$. vivax malaria in whom retroperitoneal lymphadenopathy was discovered on the initial ultrasonography of the abdomen, which disappeared while the patient was undergoing treatment. ${ }^{106}$

Acute gastroenteritis is present in roughly $15 \%$ of patients with malaria, and this presentation should be kept in mind when dealing with patients in areas endemic for malaria. Mahmood et al found nine of 108 patients with $P$. falciparum malaria had presented with acute gastroenteritis. ${ }^{107}$ Hussain et al described a 46-year-old male with malaria presenting with gastroenteritis and bloody diarrhea. ${ }^{108}$

\section{Metabolic and endocrine manifestations of malaria}

'Acidosis' in severe malaria has been attributed to a number of factors, including hypovolemia, lactic acidosis, impaired renal function, and reduced hepatic metabolism. At a $\mathrm{pH}<7.2$, basic enzyme processes and physiological mechanisms falter and, if left uncorrected, have profound effects upon myocardial contractility and arterial and venous tone, which further aggravates the impaired perfusion. In a study carried out by Maitland et al, severe acidemia (blood $\mathrm{pH}<7.2)$ was present in 96/436 $(22 \%)$ of cases, of whom $35 / 96(36 \%)$ died. ${ }^{109}$ In the absence of overt signs of dehydration, children with severe malaria have traditionally been relatively volume restricted for fear of causing pulmonary edema and aggravating raised intracranial pressure, a complication of severe malaria. However, Maitland et al found that hypovolemia is a feature of severe childhood malaria 
and a major factor in the etiology of acidosis. ${ }^{109}$ Another study carried out by the same author found that volume depletion is present at admission in the majority of children with severe malaria complicated by acidosis. ${ }^{110}$ Volume expansion in these patients corrects the hemodynamic abnormalities and is associated with improved organ function and reduction in acidosis.

Small changes in the concentration of extracellular potassium can have substantial effects on the activity of nerves and muscles. Both hypokalemia and hyperkalemia have been reported in patients with severe malaria. Hypokalemia is a common complication of severe malaria and is known to lead to cardiac arrhythmias, hypotonia, lethargy, and death. However, it is often not apparent on admission. In a study carried out by Maitland et al, out of 38 children with severe malaria and acidosis, serum potassium was normal in $31(81.6 \%)$ and low in $4(11 \%)$ children on admission. However, plasma potassium decreased rapidly within 4-8 hours of admission with 15 (40\%) patients having hypokalemia. The authors concluded that in patients with severe malaria and acidosis, correction of acidosis results in decrease in plasma potassium due to gradual intracellular shift of potassium. ${ }^{111}$ The authors also found that fractional excretion of potassium and the transtubular gradient of potassium were above normal range, indicating renal potassium loss as a cause of hypokalemia. Hyperkalemia has also been reported in patients with malaria. According to the study of Sowunmi, 7 of 51 patients with severe malaria had hyperkalemia. ${ }^{112}$ Hyperkalemia can occur in malaria due to hemolysis of red blood cells, rigors causing intense muscular contraction during febrile episodes of malaria, rhabdomyolysis, and renal failure. In severe malaria with acidosis, it is also likely that presence of acidosis contributes to the increased serum potassium through compensatory cellular exchange of hydrogen and potassium ions and through reduced renal excretion.

Hyponatremia is observed in a majority of adult and pediatric patients with severe malaria. It has been shown that mortality from hyponatremia is high, particularly when not managed promptly. A number of underlying mechanisms have been proposed, including the administration of hypotonic fluids, the syndrome of inappropriate antidiuretic hormone secretion (SIADH), cerebral salt wasting, losses through sweat and the gastrointestinal tract, renal losses, and the sick cell syndrome. ${ }^{113}$ In a study carried out by Sowunmi et al, out of 30 children with severe malaria, hyponatremia was present in $16(53 \%) .{ }^{114}$ Inappropriate secretion of arginine vasopressin (AVP) was seen in $67 \%$ of the cases. The authors concluded that high levels of AVP may contribute to the pathogenesis of hyponatremia and that in some children, fluid therapy should be cautious. In contrast to this, Hanson et al and English et al in their studies found that hypovolemia, and not SIADH, is the likely major pathophysiological mechanism, and simple rehydration with normal saline restores the plasma sodium to the normal range in the majority of the cases. ${ }^{113,115}$ Thus, a general policy of fluid restriction in children with severe malaria and hyponatremia may contribute to renal impairment. The use of hypotonic solutions when restricting fluids may exacerbate hyponatremia in those who have an appropriate antidiuretic hormone response. This may contribute to raised intracranial pressure and predispose to seizures.

'Diabetes insipidus' has been reported in association with malaria in various studies. In a study carried out by Grimwade et al, of 411 adults with malaria, 175 had severe malaria and 37 had polyuria (21\% of severe cases). Ten of $15(66 \%)$ patients investigated had urine and plasma osmolalities consistent with diabetes insipidus. The authors have emphasized that physicians should be aware of this complication. However, further studies are required to delineate the mechanism of polyuria in malaria. ${ }^{116}$ Schubert et al described a 39-year-old man with malaria who developed disseminated intravascular coagulation, polyuria, and a pituitary lesion. Empiric treatment with vasopressin improved the polyuria. ${ }^{117}$

The maintenance of plasma concentration of calcium, phosphate, and magnesium within a narrow physiological range is vital to the integrity of a variety of cellular metabolic processes. Mild asymptomatic hypocalcemia is commonly seen in approximately one-third cases of malaria regardless of the severity of infection, and a much larger proportion will experience hypocalcemia during the first few days of treatment. ${ }^{118}$ However, in some cases, hypocalcemia can be severe and symptomatic. ${ }^{119}$ Zaki et al have described a 12-year-old boy with $P$. falciparum malaria who presented with tetany. ${ }^{120}$ The serum calcium levels returned to normal with clinical recovery and parasite clearance from the blood. In a study carried out by Prabha et al, hypocalcemia was found to correlate with heavy parasitemia, complications, and Q-Tc prolongation. ${ }^{119}$ Various hypotheses have been put forward for the explanation of hypocalcemia in malaria. The main reason cited is the 'sick euparathyroid syndrome' which describes a state in which the parathyroid response to hypocalcemia is depressed during active infection, with recovery of the glandular function as the parasitemia gets cleared. ${ }^{120}$ Another hypothesis for malaria-associated hypocalcemia relates to 
the changes in phosphate metabolism. ${ }^{121} \mathrm{~A}$ lowered renal threshold for phosphate appears to be a major contributing factor for hypophosphatemia in malaria. Hypophosphatemia is associated with hypercalciuria. Hypophosphatemia can cause encephalopathy, depressed leucocyte function, increased susceptibility to Gram-negative infections, platelet dysfunction, coagulation abnormalities, and hemolytic anemia. These abnormalities are also seen in severe and complicated malaria. Reduced erythrocyte concentration of 2,3-diphosphoglycerate in hypophosphatemia can further impair the tissue oxygen delivery and vital organ function in severe malaria. ${ }^{120}$ Thus, hypophosphatemia contributes to and, in some cases, may even aggravate a variety of clinical and laboratory abnormalities associated with severe malaria. Mild asymptomatic hypomagnesemia is also known to occur in malaria. It can cause secondary hypocalcemia by impairing the release of parathormone by the parathyroid gland and through blunting the tissue response to parathormone. ${ }^{122}$ Although most studies have reported hypocalcemia as a complication of malaria, Maitland et al found hypercalcemia in $31 \%$ of the patients in their study. ${ }^{123}$ Hypocalcemia was infrequent $(<5 \%)$ at any point in their study. Intraerythrocytic calcium levels are substantially increased in PRBCs, and the authors have suggested that increase in calcium levels resulted from the release of intracellular calcium secondary to the erythrocyte lysis in malaria. This is supported by the association between hypercalcemia and severe malarial anemia, the inverse linear relationship between plasma calcium and hemoglobin levels. In addition, the presence of acidosis in severe malaria can increase the ionized calcium levels by displacing calcium from albumin.

Both hypoglycemia and hyperglycemia have been described in association with severe malaria. Decreased feeding, endotoxemia, hepatic dysfunction with impaired gluconeogenesis, and increased metabolic demand due to fever and infection can cause hypoglycemia in malaria. In their study on children with severe malaria, White et al have suggested that hypoglycemia is an important and frequent complication of severe malaria and should be suspected in any child with malaria who is comatose or convulsing. ${ }^{124}$ Dass et al have described two cases of malaria with hyperglycemia who responded well to insulin therapy. ${ }^{105}$ According to the authors, the hyperglycemia was probably due to associated sepsis or a stress response with increased counter-regulatory hormones. Both primary and secondary thyroid insufficiencies are known to occur in patients with severe malaria. Wartofsky et al studied thyroid function by a method allowing simultaneous analysis of both endogenous thyroidal release and peripheral $\mathrm{T}_{4}$ disposal in normal volunteers after induction of acute falciparum malaria. ${ }^{125}$ They found that with malarial infection, there appears to be an initial depression followed by a rebound in rates of thyroidal iodine release. In contradiction to other infections, fractional turnover and daily disposal of hormone are decreased in malaria, perhaps due to hepatic dysfunction and the consequent impairment in cellular deiodinative processes. In another study carried out by Davis et al, the pituitary-thyroid axis was assessed serially in 18 Thai adults with severe falciparum malaria and in 18 matched controls. ${ }^{126}$ They found that thyrotroph and thyroid gland function are depressed during acute, severe malaria and these changes may be an adaptation to accelerated catabolism. In addition, a raised serum cortisol could contribute to the sick euthyroid syndrome seen in malaria and other infections.

Wilson et al in their study found a raised set point for cortisol inhibition of corticotropin (ACTH) secretion, but normal ACTH responsiveness to dexamethasone in 'uncomplicated' malaria. ${ }^{127}$ A raised serum cortisol after dexamethasone in the patients might reflect the combination of a prolonged cortisol half-life and the stimulatory effects of cytokines on the adrenal cortex, with a consequent protective effect against complications such as hypoglycemia. However, in patients with 'complicated' malaria, both primary and secondary adrenal insufficiency can occur which may be attenuated by increased circulating IL-6 concentrations and impaired cortisol metabolism by the liver. Davis et al in their study found that most of the patients with severe malaria had a normal or blunted ACTH response to corticotropin-releasing hormone $(\mathrm{CRH})$ in absolute and relative terms. ${ }^{128}$ According to them, this might reflect an altered set point for cortisol inhibition of ACTH secretion analogous to that found in depression or of more globally impaired corticotroph function caused by parasitized erythrocyte sequestration within the hypothalamic-pituitary portal system or parasite production of a somatostatin-like peptide. A previous study carried out also supports this hypothesis, wherein the authors found an attenuated thyrotropin response to thyrotropin-releasing hormone in Thai patients with severe malaria. ${ }^{126}$ Thus, both the above studies suggest that there is a pituitary contribution to relative adrenal and thyroid insufficiency in severe malaria. The significance of hypothalamic-pituitary-adrenal dysfunction in malaria is illustrated by considering the patient with the lowest basal serum cortisol and a minimal response to $\mathrm{CRH}$. This patient would have a seriously impaired ability to maintain fluid and electrolyte balance and to mount a counter regulatory response to hypoglycemia. 
To assess the human reproductive endocrine response to malaria, serial serum samples were collected from 8 male and 9 female residents of Honduras infected with $P$. vivax (plus 19 male and 23 female healthy age-matched controls) and were analyzed for associations between testosterone, parasitemia, and cytokine levels. ${ }^{129}$ The study had the following results: 1) $P$. vivax infection in Honduran residents is characterized by a proinflammatory cytokine response; and 2) males infected with $P$. vivax exhibit significantly lower testosterone levels on the day of admission and significantly higher cortisol levels than age-matched healthy controls. According to the authors, whether lowered testosterone levels are the result of physiologic alterations at the level of the testis or hypothalamus is not known and will require further studies.

\section{Pregnancy and malaria}

Both $P$. vivax and $P$. falciparum are associated with severe malaria in pregnant women. In endemic areas, congenital malaria is an important cause of abortions, miscarriages, stillbirths, premature births, low birth weight, intrauterine growth retardation, and neonatal deaths. ${ }^{2}$ Low birth weight in falciparum malaria results from compromised maternofetal circulation, arising from placental sequestration of $P$. falciparum. In contrast, $P$. vivax does not seem to sequester in the placenta, although published data are limited. Placental deposition of hemozoin is notable, but inflammatory and pathological changes are otherwise modest. Systemic and local inflammatory responses and microvascular dysfunction from $P$. vivax malaria might have additional deleterious uteroplacental hemodynamic effects. ${ }^{14}$

\section{Skin manifestations in malaria}

Dermatological manifestations of malaria have been linked to both $P$. falciparum and $P$. vivax malaria. Maheshwari and Gupta have reported nine cases of malaria presenting with urticaria as the initial feature. The urticaria subsided in all these patients within $12-48 \mathrm{~h}$ of starting antimalarial treatment. Urticaria was attributed to direct effect of parasite on mast cells releasing histamine, involvement of complement system, and intense elevation of $\operatorname{IgG}$ and $\mathrm{IgM}$ antibodies. $^{130}$

\section{Malnutrition and malaria}

Malnutrition plays a major role in child mortality and is associated with lasting effects on growth and cognitive development. The relationship between malnutrition and $P$. falciparum malaria is complex. According to some studies, falciparum malaria results in malnutrition, and on the other hand, some mention that malnutrition may protect against $P$. falciparum malaria. In a study carried out by Williams et al, malnutrition was significantly associated with $P$. vivax malaria. $P$. vivax being a potent stimulus for TNF secretion (which induces both anorexia and cachexia) can cause malnutrition. ${ }^{131}$ In addition, the deleterious catabolic responses associated with chronic relapsing-remitting inflammation probably contribute to malnutrition. ${ }^{14}$ Thus, malaria can result in chronic relapsing-remitting debilitating fever, hypoproteinemia, edema, and kwashiorkor-like weight loss.

\section{Conclusion}

Malaria is a multisystem disorder which can mimic many diseases. Physicians, especially those in endemic areas, should be aware of the varied manifestations and maintain a high index of suspicion for the disease so that the diagnosis and treatment are timely and morbidity and mortality minimized.

\section{Disclosure}

The authors report no conflicts of interest in this work.

\section{References}

1. Greenwood B, Mutabingwa T. Malaria in 2002. Nature. 2002;415(6872): 670-672.

2. Krause PJ. Malaria (Plasmodium). In: Behrman RE, Kliegman RM, Jenson HB, editors. Nelson Textbook of Pediatrics. 18th ed. Philadelphia, PA: WB Saunders; 2007:1477-1485.

3. Rao A, Kumar MK, Joseph T, Bulusu G. Cerebral malaria: insights from host-parasite protein-protein interactions. Malar J. 2010; 9:155.

4. Sharma A, Khanduri U. How benign is benign tertian malaria? J Vector Borne Dis. 2009;46(2):141-144.

5. Islam N, Qamruddin K. Unusual complications in benign tertian malaria. Trop Geogr Med. 1995;47(3):141-143.

6. Makkar RP, Monga SM, Gupta AK. Plasmodium vivax malaria presenting with severe thrombocytopenia. Braz J Infect Dis. 2002;6(5): 263-265.

7. Nautiyal A, Singh S, Parameswaran G, DiSalle M. Hepatic dysfunction in a patient with Plasmodium vivax infection. MedGenMed. 2005;7(1):8

8. Prakash J, Singh AK, Kumar NS, Saxena RK. Acute renal failure in Plasmodium vivax malaria. J Assoc Physicians India. 2003;51: 265-267.

9. Beg MA, Khan R, Baig SM, Gulzar Z, Hussain R, Smego RA Jr. Cerebral involvement in benign tertian malaria. Am J Trop Med Hyg. 2002;67(3): 230-232.

10. Lomar AV, Vidal JE, Lomar FP, Barbas CV, de Matos GJ, Boulos M. Acute respiratory distress syndrome due to vivax malaria: case report and literature review. Braz J Infect Dis. 2005;9(5):425-430.

11. Singh UK, Kumar R, Sharma VK. Increased urinary frequency as a presentation of Plasmodium falciparum malaria. Pediatr Infect Dis J. 1994; 13(11):1024

12. Miller LH, Baruch DI, Marsh K, Doumbo OK. The pathogenic basis of malaria. Nature. 2002;415(6872):673-679. 
13. Mohanty S, Patel DK, Pati SS, Mishra SK. Adjuvant therapy in cerebral malaria. Indian J Med Res. 2006;124(3):245-260.

14. Anstey NM, Russell B, Yeo TW, Price RN. The pathophysiology of vivax malaria. Trends Parasitol. 2009;25(5):220-227.

15. Crabb BS, Cowman AF. Plasmodium falciparum virulence determinants unveiled. Genome Biol. 2002;3(11):REVIEWS1031.

16. Garg RK, Karak B, Misra S. Neurological manifestations of malaria: an update. Neurol India. 1999;47(2):85-91.

17. Clark IA, Alleva LM, Mills AC, Cowden WB. Pathogenesis of malaria and clinically similar conditions. Clin Microbiol Rev. 2004;17(3): 509-539.

18. Newton CR, Hien TT, White N. Cerebral malaria. J Neurol Neurosurg Psychiatry. 2000;69(4):433-441.

19. Waruiru CM, Newton CR, Forster D, et al. Epileptic seizures and malaria in Kenyan children. Trans R Soc Trop Med Hyg. 1996;90(2): 152-155.

20. Phillips RE, Solomon T. Cerebral malaria in children. Lancet. 1990; 336(8727):1355-1360.

21. Mishra SK, Newton CR. Diagnosis and management of the neurological complications of falciparum malaria. Nat Rev Neurol. 2009;5(4): 189-198.

22. Gall C, Spuler A, Fraunberger P. Subarachnoid hemorrhage in a patient with cerebral malaria. N Engl J Med. 1999;341(8):611-613.

23. Mathur SL, Hakim A, Lodha R, Chowdhry P, Jain R. Subarachnoid haemorrhage in falciparum malaria: an unreported presentation. J Assoc Physicians India. 1992;40(5):348.

24. Dwarakanath S, Suri A, Mahapatra AK. Spontaneous subdural empyema in falciparum malaria: a case study. J Vector Borne Dis. 2004; 41(3-4):80-82.

25. Das CJ, Sharma R. Central pontine myelinolysis in a case of cerebral malaria. Br J Radiol. 2007;80(960):e293-e295.

26. Blocker WW, Kastl AJ, Daroff RB. The psychiatric manifestations of cerebral malaria. Am J Psychiatry. 1968;125:192-196.

27. Arun Prakash MV, Stein G. Malaria presenting as atypical depression. Br J Psychiatry. 1990;156:594-595.

28. Kochar DK, Shubhakaran, Kumawat B. Neuropsychiatric manifestations in malaria. J Assoc Physicians India. 1998;46:886-890.

29. Thapa R, Biswas B. Childhood mefloquine-induced mania and psychosis: a case report. J Child Neurol. 2009;24(8):1008-1009.

30. Sahoo S, Kumar M, Sinha VK. Chloroquine-induced recurrent psychosis. Am J Ther. 2007;14(4):406-407.

31. Onifade EU, Danesi MA. Acute cerebellar ataxia associated with falciparum malaria in Nigerian patients. Niger Postgrad Med J. 2004; 11(2):140-144.

32. Mohapatra MK, Panda BK, Das SP. Cerebellar ataxia in falciparum malaria - a report of two cases. Indian J Malariol. 2000;37(1-2): 46-48.

33. Bhandari K, Bhandari P. Cerebellar syndrome in malaria. Indian Pediatr. 1989;26(10):1037-1038.

34. Nguyen TH, Day NP, Ly VC, et al. Post-malaria neurological syndrome. Lancet. 1996;348(9032):917-921.

35. Schnorf H, Diserens K, Schnyder H, et al. Corticosteroid-responsive postmalaria encephalopathy characterized by motor aphasia, myoclonus, and postural tremor. Arch Neurol. 1998;55(3):417-420.

36. Lawn SD, Flanagan KL, Wright SG, Doherty TF, GodfreyFaussett P. Postmalaria neurological syndrome: two cases from the Gambia. Clin Infect Dis. 2003;36(2):e29-e31.

37. Prendki V, Elzière C, Durand R, et al. Post-malaria neurological syndrome - two cases in patients of African origin. Am J Trop Med Hyg. 2008;78(5):699-701.

38. Wijesundere A. Guillain-Barré syndrome in Plasmodium falciparum malaria. Postgrad Med J. 1992;68(799):376-377.

39. Sokrab TE, Eltahir A, Idris MN, Hamid M. Guillain-Barré syndrome following acute falciparum malaria. Neurology. 2002;59(8): 1281-1283.

40. Kanjalkar M, Karnad DR, Narayana RV, Shah PU. Guillain-Barre syndrome following malaria. J Infect. 1999;38(1):48-50.
41. Kochar DK, Joshi A, Agarwal N, Shubhkaran, Thanvi I. Hemiparesis: an uncommon complication of falciparum malaria. J Assoc Physicians India. 1999;47(11):1108-1109.

42. Van Hensbroek MB, Palmer A, Jaffar S, Schneider G, Kwiatkowski D. Residual neurologic sequelae after childhood cerebral malaria. J Pediatr. 1997;131(1 Pt 1):125-129.

43. Kochar DK, Shubhakaran, Kumawat BL, et al. Cerebral malaria in Indian adults: a prospective study of 441 patients from Bikaner, northwest India. J Assoc Physicians India. 2002;50:234-241.

44. Carter JA, Neville BG, White S, et al. Increased prevalence of epilepsy associated with severe falciparum malaria in children. Epilepsia. 2004;45(8):978-981.

45. Aleem MA. Epilepsy in malaria. Epilepsia. 2005;46(4):601. Author reply 601-602.

46. Beare NA, Taylor TE, Harding SP, Lewallen S, Molyneux ME. Malarial retinopathy: a newly established diagnostic sign in severe malaria. Am J Trop Med Hyg. 2006;75(5):790-797.

47. Maude RJ, Dondorp AM, Abu Sayeed A, Day NP, White NJ, Beare NA. The eye in cerebral malaria: what can it teach us? Trans $R$ Soc Trop Med Hyg. 2009;103(7):661-664.

48. Kale VP, Bichile LS, Bajpai S. Falciparum malaria induced retrobulbar neuritis. J Postgrad Med. 2004;50(2):150.

49. Mishra SK, Pati SS, Mahanta KC, Mohanty S. Rhabdomyolysis in falciparum malaria-a series of twelve cases (five children and seven adults). Trop Doct. 2010;40(2):87-88.

50. Miller KD, White NJ, Lott JA, Roberts JM, Greenwood BM. Biochemical evidence of muscle injury in African children with severe malaria. J Infect Dis. 1989;159(1):139-142.

51. Senanayake N, Wimalawansa SJ. Periodic paralysis complicating malaria. Postgrad Med J. 1981;57(667):273-274.

52. Haldar K, Mohandas N. Malaria, erythrocytic infection, and anemia. Hematology Am Soc Hematol Educ Program. 2009:87-93.

53. Ohnishi K, Mitsui K, Komiya N, Iwasaki N, Akashi A, Hamabe Y. Clinical case report: falciparum malaria with hemophagocytic syndrome. Am J Trop Med Hyg. 2007;76(6):1016-1018.

54. Aouba A, Noguera ME, Clauvel JP, Quint L. Haemophagocytic syndrome associated with plasmodium vivax infection. Br J Haematol. 2000;108(4):832-833.

55. Irving KG, Kemp E, Olivier BJ, Mendelow BV. Unusual presentation of malaria as a leukaemoid reaction. A case report. S Afr Med J. 1987; 71(9):597-598.

56. Soe $\mathrm{S}$, Tin $\mathrm{S}$, Than $\mathrm{S}$. Myeloid leukemoid reaction in malaria infection. Southeast Asian J Trop Med Public Health. 1991;22(4):677-678.

57. Sharma SK, Das RK, Das BK, Das PK. Haematological and coagulation profile in acute falciparum malaria. J Assoc Physicians India. 1992; 40(9):581-583.

58. Modiano D, Sirima BS, Konaté A, Sanou I, Sawadogo A. Leucocytosis in severe malaria. Trans R Soc Trop Med Hyg. 2001;95(2):175-176.

59. Aggarwal A, Rath $S$, Shashiraj. Plasmodium vivax malaria presenting with severe thrombocytopenia. J Trop Pediatr. 2005;51:120-121.

60. Katira B, Shah I. Thrombocytopenia in Plasmodium vivax infected children. J Vector Borne Dis. 2006;43(3):147-149.

61. Lacerda MV, Alexandre MA, Santos PD, Arcanjo AR, Alecrim WD, Alecrim MG. Idiopathic thrombocytopenic purpura due to vivax malaria in the Brazilian Amazon. Acta Trop. 2004;90(2):187-190.

62. Krishnan A, Karnad DR, Limaye U, Siddharth W. Cerebral venous and dural sinus thrombosis in severe falciparum malaria. $J$ Infect. 2004; 48(1):86-90.

63. Luvira V, Chamnanchanunt S, Thanachartwet V, Phumratanaprapin W, Viriyavejakul A. Cerebral venous sinus thrombosis in severe malaria. Southeast Asian J Trop Med Public Health. 2009;40(5):893-897.

64. Mohapatra MK, Khan MA. Sternal tenderness in falciparum malaria. J Assoc Physicians India. 1996;44(9):667.

65. Das BS. Renal failure in malaria. J Vector Borne Dis. 2008;45(2): 83-97.

66. Barsoum RS. Malarial acute renal failure. J Am Soc Nephrol. 2000; 11(11):2147-2154. 
67. Barsoum RS. Malarial nephropathies. Nephrol Dial Transplant. 1998; 13(6):1588-1597.

68. Prakash J, Singh AK, Gujrati S, Maheshwari A. Acute renal failure in malaria: changing trends. Indian J Nephrol. 2002;12(4):113-117.

69. Boonpucknavig V, Sitprija V. Renal disease in acute Plasmodium falciparum infection in man. Kidney Int. 1979;16(1):44-52.

70. David J, Shanbag P, More V. Plasmodium vivax malaria presenting as the nephrotic syndrome in an infant. Trop Doct. 2009;39(2):127-128.

71. Sanghai SR, Shah I. Plasmodium vivax with acute glomerulonephritis in an 8-year old. J Vector Borne Dis. 2010;47(1):65-66.

72. Sharma J, Bharadawa K, Shah K, Dave S. Plasmodium vivax malaria presenting as hemolytic-uremic syndrome. Indian Pediatr. 1993;30(3): 369-371.

73. Dogra PN, Wadhwa SN, Mehta VK. Vivax malaria causing priapism. J Assoc Physicians India. 1991;39(7):571-572.

74. Sinha S, Goel AK, Kumar A, Chattopadhyay TK. Priapism in malaria. J Assoc Physicians India. 1992;40(11):769.

75. De Silva HJ, Herath S, de Silva NR. Urinary frequency in falciparum malaria. Trans R Soc Trop Med Hyg. 1992;86(2):137.

76. Anstey NM, Jacups SP, Cain T, et al. Pulmonary manifestations of uncomplicated falciparum and vivax malaria: cough, small airways obstruction, impaired gas transfer, and increased pulmonary phagocytic activity. J Infect Dis. 2002;185(9):1326-1334.

77. Maguire GP, Handojo T, Pain MC, et al. Lung injury in uncomplicated and severe falciparum malaria: a longitudinal study in Papua, Indonesia. J Infect Dis. 2005;192(11):1966-1974.

78. Mohan A, Sharma SK, Bollineni S. Acute lung injury and acute respiratory distress syndrome in malaria. J Vector Borne Dis. 2008;45(3): 179-193.

79. Fein IA, Rackow EC, Shapiro L. Acute pulmonary edema in Plasmodium falciparum malaria. Am Rev Respir Dis. 1978;118(2): 425-429.

80. Munteis E, Mellibovsky L, Márquez MA, Mínguez S, Vázquez E, Díez A. Pulmonary involvement in a case of Plasmodium vivax malaria. Chest. 1997;111(3):834-835.

81. Yale SH, Adlakha A, Sebo TJ, Ryu JH. Bronchiolitis obliterans organizing pneumonia caused by Plasmodium vivax malaria. Chest. 1993;104(4):1294-1296.

82. Taylor WR, Cañon V, White NJ. Pulmonary manifestations of malaria: recognition and management. Treat Respir Med. 2006;5(6): 419-428.

83. Sirivichayakul C, Chanthavanich P, Chokejindachai W, Pengsaa K, Kabkaew K, Saelim R. Pleural effusion in childhood falciparum malaria Southeast Asian J Trop Med Public Health. 2000;31(1):187-189.

84. Franzen D, Curtius JM, Heitz W, Höpp HW, Diehl V, Hilger HH. Cardiac involvement during and after malaria. Clin Investig. 1992;70(8): 670-673.

85. Kohli R, Gulati S, Kapoor A, Singh S. Cardiac tamponade: an unusual complication of Plasmodium falciparum-induced acute renal failure. Nephrol Dial Transplant. 2003;18(8):1678-1679.

86. Günther A, Grobusch MP, Slevogt H, Abel W, Burchard GD. Myocardial damage in falciparum malaria detectable by cardiac troponin T is rare. Trop Med Int Health. 2003;8(1):30-32.

87. Sharma SN, Mohapatra AK, Machave YV. Chronic falciparum cardiomyopathy. J Assoc Physicians India. 1987;35(3):251-252.

88. Mohapatra MK, Mohanty NK, Das SP. Myocardial injury: an unrecognized complication of cerebral malaria. Trop Doct. 2000;30(3): 188-189.

89. Yacoub S, Lang HJ, Shebbe M, et al. Cardiac function and hemodynamics in Kenyan children with severe malaria. Crit Care Med. 2010; 38(3):940-945.

90. Song JY, Park CW, Jo YM, et al. Two cases of Plasmodium vivax malaria with the clinical picture resembling toxic shock. Am J Trop Med Hyg. 2007;77(4):609-611.

91. Ghafoor SZ, MacRae EA, Harding KG, Patel GK. Symmetrical peripheral digital gangrene following severe Plasmodium falciparum malaria-induced disseminated intravascular coagulopathy. Int Wound J. 2010;7(5):418-422.
92. Liechti ME, Zumsteg V, Hatz CF, Herren T. Plasmodium falciparum cerebral malaria complicated by disseminated intravascular coagulation and symmetrical peripheral gangrene: case report and review. Eur J Clin Microbiol Infect Dis. 2003;22(9):551-554.

93. Chaudhary SC, Kumar V, Gupta A. Purpura fulminans: a rare presentation of a common disease. Trop Doct. 2010;40(4):238-239.

94. Mocumbi AO, Songane M, Salomão C, Ulibarri R, Ferreira MB, Yacoub MH. Lack of evidence of myocardial damage in children with plasmodium falciparum severe and complicated malaria from an endemic area for endomyocardial fibrosis. J Trop Pediatr. 2009 Nov 30. Epub ahead of print.

95. Bhalla A, Suri V, Singh V. Malarial hepatopathy. J Postgrad Med. 2006;52(4):315-320.

96. Kochar DK, Agarwal P, Kochar SK, et al. Hepatocyte dysfunction and hepatic encephalopathy in Plasmodium falciparum malaria. QJM. 2003;96(7):505-512.

97. Devarbhavi H, Alvares JF, Kumar KS. Severe falciparum malaria simulating fulminant hepatic failure. Mayo Clin Proc. 2005;80(3):355-358.

98. Davenport M. Neonatal malaria and obstructive jaundice. Arch Dis Child. 1986;61(5):515-517.

99. Ozsoy MF, Oncul O, Pekkafali Z, Pahsa A, Yenen OS. Splenic complications in malaria: report of two cases from Turkey. $J$ Med Microbiol. 2004;53(Pt 12):1255-1258.

100. Yagmur Y, Kara IH, Aldemir M, Büyükbayram H, Tacyildiz IH, Keles C. Spontaneous rupture of malarial spleen: two case reports and review of literature. Crit Care. 2000;4(5):309-313.

101. Agarwal VK, Agarwal S, Pathak T. Splenic infarct in falciparum malaria. Indian Pediatr. 1997;34(11):1050-1051.

102. Contini S, Lewis HR. Spleen abscess as malaria complication. Emerg Infect Dis. 2006;12(3):529-531.

103. Kumar S, Jain AP, Vikas. Falciparum malaria presenting as acute pancreatitis. J Vector Borne Dis. 2010;47(2):121-122.

104. Kuttiat VS, Kohli U. Acute acalculous cholecystitis associated with malarial infection in children: report of two cases. J Trop Pediatr. 2007;53(1):59-61.

105. Dass R, Barman H, Duwarah SG, Deka NM, Jain P, Choudhury V. Unusual presentations of malaria in children: an experience from a tertiary care center in North East India. Indian J Pediatr. 2010;77(6): $655-660$

106. Sood A, Midha V. Abdominal lymphadenopathy in malaria. Clin Infect Dis. 1999;28(2):400-401.

107. Mahmood K, Jairamani KL, Abbasi B, et al. Falciparum malaria: various presentations. Pak J Med Sci. 2006;22(3):234-237.

108. Hussain WM, Bukhari SZ, Fatani MI, Karima TM, Madani TA, Badreddine S. Misdiagnosis of an imported case of malaria caused by Plasmodium falciparum. J Infect Dev Ctries. 2009;3(2): $112-114$

109. Maitland K, Levin M, English M, et al. Severe P. falciparum malaria in Kenyan children: evidence for hypovolaemia. QJM. 2003;96(6):427-434

110. Maitland K, Pamba A, Newton CR, Levin M. Response to volume resuscitation in children with severe malaria. Pediatr Crit Care Med. 2003;4(4):426-431.

111. Maitland K, Pamba A, Newton CR, Lowe B, Levin M. Hypokalemia in children with severe falciparum malaria. Pediatr Crit Care Med. 2004;5(1):81-85.

112. Sowunmi A. Clinical study of cerebral malaria in African children. Afr J Med Med Sci. 1997;26(1-2):9-11.

113. Hanson J, Hossain A, Charunwatthana P, et al. Hyponatremia in severe malaria: evidence for an appropriate anti-diuretic hormone response to hypovolemia. Am J Trop Med Hyg. 2009;80(1):141-145.

114. Sowunmi A, Newton CR, Waruiru C, Lightman S, Dunger DB. Arginine vasopressin secretion in Kenyan children with severe malaria. J Trop Pediatr. 2000;46(4):195-199.

115. English MC, Waruiru C, Lightowler C, Murphy SA, Kirigha G, Marsh K. Hyponatraemia and dehydration in severe malaria. Arch Dis Child. 1996;74(3):201-205. 
116. Grimwade K, French N, Mthembu D, Gilks C. Polyuria in association with Plasmodium falciparum malaria in a region of unstable transmission. Trans R Soc Trop Med Hyg. 2004;98(4):255-260.

117. Schubert S, Achenbach H, Engelmann L, Borte G, Stumvoll M, Koch CA. Central diabetes insipidus in a patient with malaria tropica. J Endocrinol Invest. 2006;29(3):265-266.

118. Davis TM, Li GQ, Guo XB, Spencer JL, St John A. Serum ionized calcium, serum and intracellular phosphate, and serum parathormone concentrations in acute malaria. Trans R Soc Trop Med Hyg. 1993; 87(1):49-53.

119. Prabha MR, Pereira P, Chowta N, Hegde BM. Clinical implications of hypocalcemia in malaria. Indian J Med Res. 1998;108:62-65.

120. Zaki SA, Shanbag P, Shenoy P. Unusual presentation of malaria as tetany: a case report. J Vector Borne Dis. 2010;47(2):123-125.

121. Davis TM, Pukrittayakamee S, Woodhead JS, Holloway P, Chaivisuth B, White NJ. Calcium and phosphate metabolism in acute falciparum malaria. Clin Sci (Lond). 1991;81(3):297-304.

122. Greenbaun LA. Electrolytes and acid-base disorders. In: Behrman RE, Kliegman RM, Jenson HB, editors. Nelson Textbook of Pediatrics. 17th ed. Philadelphia, PA: WB Saunders; 2004:267-308.

123. Maitland K, Pamba A, Fegan G, et al. Perturbations in electrolyte levels in Kenyan children with severe malaria complicated by acidosis. Clin Infect Dis. 2005;40(1):9-16.

124. White NJ, Miller KD, Marsh K, et al. Hypoglycaemia in African children with severe malaria. Lancet. 1987;1(8535):708-711.
125. Wartofsky L, Martin D, Earll JM. Alterations in thyroid iodine release and the peripheral metabolism of thyroxine during acute falciparum malaria in man. J Clin Invest. 1972;51(9):2215-2232.

126. Davis TM, Supanaranond W, Pukrittayakamee S, et al. The pituitarythyroid axis in severe falciparum malaria: evidence for depressed thyrotroph and thyroid gland function. Trans $R$ Soc Trop Med Hyg. 1990;84(3):330-335.

127. Wilson M, Davis TM, Binh TQ, Long TT, Danh PT, Robertson K. Pituitary-adrenal function in uncomplicated falciparum malaria. Southeast Asian J Trop Med Public Health. 2001;32(4):689-695.

128. Davis TM, Li TA, Tran QB, et al. The hypothalamic-pituitaryadrenocortical axis in severe falciparum malaria: effects of cytokines. J Clin Endocrinol Metab. 1997;82(9):3029-3033.

129. Muehlenbein MP, Alger J, Cogswell F, James M, Krogstad D. The reproductive endocrine response to Plasmodium vivax infection in Hondurans. Am J Trop Med Hyg. 2005;73(1):178-187.

130. Maheshwari RK, Gupta BD. Urticaria in malaria. Indian Pediatr. 1984;21(8):663.

131. Williams TN, Maitland K, Phelps L, et al. Plasmodium vivax: a cause of malnutrition in young children. QJM. 1997;90(12):751-757.
Research and Reports in Tropical Medicine

\section{Publish your work in this journal}

Research and Reports in Tropical Medicine is an international, peerreviewed, open access journal publishing original research, case reports, editorials, reviews and commentaries on all areas of tropical medicine, including: Diseases and medicine in tropical regions; Entomology; Epidemiology; Health economics issues; Infectious disease; Laboratory

\section{Dovepress}

science and new technology in tropical medicine; Parasitology; Public health medicine/health care policy in tropical regions; and Microbiology. The manuscript management system is completely online and includes a very quick and fair peer-review system. Visit http://www.dovepress. com/testimonials.php to read real quotes from published authors. 